\title{
Linearized gravity on the Randall-Sundrum two-brane background with curvature terms in the action for the branes
}

\author{
Yuri Shtanov $^{a *}$ and Alexander Viznyuk ${ }^{a, b \dagger}$ \\ ${ }^{a}$ Bogolyubov Institute for Theoretical Physics, Kiev 03143, Ukraine \\ ${ }^{b}$ Department of Physics, Taras Shevchenko National University, Kiev 03022, Ukraine
}

\begin{abstract}
We study gravitational perturbations in the Randall-Sundrum two-brane background with scalar-curvature terms in the action for the branes, allowing for positive as well as negative bulk gravitational constant. In the zero-mode approximation, we derive the linearized gravitational equations, which have the same form as in the original Randall-Sundrum model but with different expressions for the effective physical constants. We develop a generic method for finding tachyonic modes in the theory, which, in the model under consideration, may exist only if the bulk gravitational constant is negative. In this case, if both brane gravitational constants are nonzero, the theory contains one or two tachyonic mass eigenvalues in the gravitational sector. If one of the brane gravitational constants is set to zero, then either a single tachyonic mass eigenvalue is present or tachyonic modes are totally absent depending on the relation between the nonzero brane gravitational constant and brane separation. In the case of negative bulk gravitational constant, the massive gravitational modes have ghost-like character, while the massless gravitational mode is not a ghost in the case where tachyons are absent.
\end{abstract}

\section{INTRODUCTION}

Linear gravitational perturbations of the flat braneworld models were studied beginning from the seminal papers by Randall \& Sundrum [1,2], where their spectrum was shown to contain, besides the zero mode, also an infinite tower of Kaluza-Klein massive modes. Since then, perturbations in various types of braneworld scenarios and in various approximations were considered in the flat case as well as on the cosmological background (which is a much more complicated issue still far from being well understood; see [3] and references therein). In this paper, we study certain aspects of linear perturbations on a particular simple background, which, to our knowledge, have not been previously investigated. We consider the Randall-Sundrum two-brane model (the so-called RS1 model [1]) supplemented by scalarcurvature terms in the action for both branes. Historically, the induced scalar-curvature

\footnotetext{
*E-mail: shtanov@bitp.kiev.ua

†E-mail: viznyuk@bitp.kiev.ua
} 
term for the brane was introduced in [4] (see also [5]) as a method of making gravity on the brane effectively four-dimensional even in the flat infinite bulk space. The corresponding cosmological models were initiated in $[5,6]$. The effects of these scalar-curvature terms in the two-brane setup under consideration were recently studied in [7-11]. Perturbations in the one-brane (RS2 [2]) counterpart of this model were previously investigated in [12,13].

The study of the braneworld models is usually confined to the case of positive bulk and brane gravitational constants. (The signs of the gravitational constants in this case are defined relative to the signs of the conventional matter Lagrangians on the branes.) This can be explained by the fact that only positive values of the bulk gravitational constant are allowed in the Randall-Sundrum model, since its sign coincides with the sign of the effective Newton's constant in that model $[1,2]$. When one adds curvature terms in the action for the branes, this assumption might be relaxed, and, in this paper, we allow for positive as well as negative bulk gravitational constant while keeping gravitational constants on the branes positive. More generally, one could consider various relations between the signs of the four-dimensional gravitational constants in the action for the branes, bulk gravitational constant, and conventional matter Lagrangians [8-11]. Although we are not aware of any fundamental multidimensional theory that can produce different signs of the gravitational couplings for the bulk and branes, we consider this possibility from the viewpoint of the effective action regardless of the unknown underlying theory.

The study of both signs of the bulk gravitational constant is partially motivated by the existence of braneworld models with interesting behaviour that require negative brane tension. One of them is the model of disappearing dark energy (DDE) [14,15]. The DDE model is a braneworld model of expanding universe which, after the current period of acceleration, re-enters the matter-dominated regime continuing indefinitely in the future. The merit of this model of dark energy is the absence of the cosmological event horizon owing to the fact that the universe becomes flat, rather than De Sitter, in the asymptotic future. The DDE model is based on the generic braneworld action with the bulk cosmological constant and brane tension satisfying the Randall-Sundrum constraint, and also including the curvature term in the action for the brane. For the consistency of this model with the current cosmological observations (specifically, for the condition $\Omega_{\mathrm{m}}<1$ on the dark-matter cosmological parameter), the brane tension has to be negative [14]. Negative brane tension is required also for the existence of unusual 'quiescent' singularities [16] in the AdS-embedded braneworld models, which occur during the universe expansion and are characterized by finiteness of the scale factor, Hubble parameter, and matter density.

The bulk gravitational constant enters the homogeneous cosmological equations on the brane in even power; therefore, its sign does not matter on the level of the homogeneous cosmology on the brane [14]. However, the relation between the sign of the bulk gravitational constant and the sign of the brane tension is of importance for the small-scale gravitational physics in the braneworld, in particular, for the behaviour of cosmological perturbations. This can be seen already from the fact that the property of 'localization' of five-dimensional gravity in the neighbourhood of the brane (that the warp factor locally decreases as one moves away from the brane) requires the brane tension and the bulk gravitational constant to be of the same sign. This localization property may turn out to be important for a consistent braneworld theory and, therefore, for negative-tension branes, it may require negative gravitational constant in the bulk. Thus, it seems important to keep open this 
possibility when generalizing the Randall-Sundrum model by including the curvature terms in the action for the branes.

The issue of ghosts in a theory with positive bulk gravitational constant but arbitrary signs of the brane gravitational constants was recently under investigation in [9], and certain regions of parameters were ruled out. In this paper, we consider somewhat complimentary situation where the brane gravitational constants are always positive with respect to the conventional matter Lagrangian, while the bulk gravitational constant can be of any sign. ${ }^{1}$ We will be mainly concerned with the issue of tachyons in the theory of this kind, which, to our knowledge, was not discussed in the literature before. Our original method of finding tachyonic modes in the two-brane background is quite general and can be applied to other braneworld models leading to the same system of equations for the bulk gravitational modes, in particular, to the model with the Gauss-Bonnet action in the bulk considered in [8].

It will be shown below that, unlike in the pure Randall-Sundrum case, the presence of the curvature terms in the action for the branes leads to a possibility of unwanted tachyonic modes if the bulk gravitational constant is negative. We demonstrate that, in this case, there can be only one or two tachyonic mass eigenvalues in the theory under consideration and determine the range of parameters for which tachyonic modes do not exist. We will also see that the massive gravitational modes have ghost-like character in the case of negative bulk gravitational constant, while the massless gravitational mode is not a ghost in the case where tachyons are absent. These results are in agreement with those of [9]. Thus, it is mainly the presence of ghosts in the Kaluza-Klein massive spectrum of gravity that makes models with negative bulk gravitational constant problematic.

This paper is organized in the following manner. After describing the model, we review a suitable theory of linear gravitational perturbations on the Randall-Sundrum two-brane background. Then, in the zero-mode approximation, we derive the linearized system of gravitational equations with matter confined to the visible and/or hidden brane. These equations have the same form as in the original RS1 model but with different physical constants. After that, we specially investigate the case of negative bulk gravitational constant and show that the linearized theory can contain tachyonic gravitational modes. In this case, one or two tachyonic mass eigenvalues are observed if both brane gravitational constants are nonzero. If one of the brane gravitational constants is zero, then either a single tachyonic mass eigenvalue is present or tachyonic modes are totally absent; which of these two possibility is realized depends upon the values of the nonzero brane gravitational constant and brane separation. We determine the range of parameters for which tachyonic modes are present or absent. Following [9], we also consider the ghost modes for the radion and graviton in our theory. In the case of negative bulk gravitational constant, the range of parameters where the radion is ghost free is rather narrow, and all massive gravitational modes have ghost-like nature.

We also calculate the effective gravitational potentials of static matter sources on the visible and hidden brane in the generic case.

\footnotetext{
${ }^{1}$ The results obtained in one of such theories are usually easy to apply to another theory by using the change of the overall sign of the action.
} 


\section{THE MODEL}

The action of the model in the neighbourhood of one brane has the form

$$
S=M^{3}\left[\int_{\text {bulk }}(\mathcal{R}-2 \Lambda)-2 \int_{\text {brane }} K\right]+\int_{\text {brane }}\left(m^{2} R-2 \sigma\right)+\int_{\text {brane }} L\left(h_{a b}, \phi\right),
$$

where the first part, proportional to the cube of the bulk Planck mass $M^{3}$, describes the bulk bounded by the brane, and the remaining integrals are taken over the brane. Here, $\mathcal{R}$ is the curvature scalar in the bulk, $R$ is the curvature scalar of the induced metric $h_{a b}$ on the brane, $K$ is the trace of the tensor of the intrinsic curvature $K_{a b}$ of the brane with respect to the inner normal, $L\left(h_{a b}, \phi\right)$ is the Lagrangian of the matter fields $\phi$ on the brane, and integration in (1) implies natural volume elements in the bulk and on the brane. The action is similar in the neighbourhood of the other brane. In principle, two branes in our model may have different Planck masses $m$, and, to allow for solutions with flat vacuum branes, their tensions $\sigma$ must have opposite signs and satisfy the well-known constraint $[1,2]$

$$
\Lambda_{\mathrm{RS}} \equiv \frac{\Lambda}{2}+\frac{\sigma^{2}}{3 M^{6}}=0
$$

Note that, in this paper, we allow for positive as well as negative signs of the bulk Planck mass parameter $M$, the consequences of which will become clear later.

Action (1) leads to the bulk described by the usual Einstein equation with cosmological constant:

$$
\mathcal{G}_{a b}+\Lambda g_{a b}=0
$$

while the field equation on the brane is

$$
m^{2} G_{a b}+\sigma h_{a b}=\tau_{a b}+M^{3}\left(K_{a b}-h_{a b} K\right)
$$

where $\tau_{a b}$ is the stress-energy tensor on the brane stemming from the last term in action (1).

By contracting the Gauss identity

$$
R_{a b c}{ }^{d}=h_{a}{ }^{f} h_{b}{ }^{g} h_{c}{ }^{k} h^{d}{ }_{j} \mathcal{R}_{f g k}{ }^{j}+K_{a c} K_{b}{ }^{d}-K_{b c} K_{a}{ }^{d}
$$

on the brane and using Eq. (3), one obtains the 'constraint' equation

$$
R-2 \Lambda+K_{a b} K^{a b}-K^{2}=0
$$

which, together with (4), implies the following closed scalar equation on the brane:

$$
M^{6}(R-2 \Lambda)+\left(m^{2} G_{a b}+\sigma h_{a b}-\tau_{a b}\right)\left(m^{2} G^{a b}+\sigma h^{a b}-\tau^{a b}\right)-\frac{1}{3}\left(m^{2} R-4 \sigma+\tau\right)^{2}=0
$$

where $\tau=h^{a b} \tau_{a b}$.

In the case of a vacuum brane $\left(\tau_{a b}=0\right)$, Eq. (7) takes the form

$$
\left(M^{6}+\frac{2}{3} \sigma m^{2}\right) R+m^{4}\left(R_{a b} R^{a b}-\frac{1}{3} R^{2}\right)-4 M^{6} \Lambda_{\mathrm{RS}}=0
$$


where $\Lambda_{\mathrm{RS}}$ is given by Eq. (2). It should be noted that the second term in Eq. (8) has precisely the form of one of the terms in the expression for the conformal anomaly, which describes the vacuum polarization at the one-loop level in curved space-time (see, e.g., [17]). ${ }^{2}$

Another useful relation is the Codazzi identity

$$
D_{a}\left(K_{b}^{a}-h_{b}^{a} K\right)=0,
$$

which is valid at any timelike hypersurface in the bulk, in particular, on the branes, due to Eq. (3). Here, $D_{a}$ denotes the unique covariant derivative on the timelike hypersurface associated with the induced metric $h_{a b}$.

The gravitational equations in the bulk can be integrated by using Gaussian normal coordinates, as described, e.g., in [18]. Specifically, in the Gaussian normal coordinates $(x, y)$, where $x=\left\{x^{\alpha}\right\}$ are the coordinates on the brane and $y$ is the fifth coordinate in the bulk, the metric is written as

$$
d s^{2}=d y^{2}+h_{\alpha \beta}(x, y) d x^{\alpha} d x^{\beta} .
$$

Introducing also the tensor of extrinsic curvature $K_{a b}$ of every hypersurface $y=$ const, one can obtain the following system of differential equations for the components $h_{\alpha \beta}$ and $K^{\alpha}{ }_{\beta}$ :

$$
\begin{gathered}
\frac{\partial K^{\alpha}{ }_{\beta}}{\partial y}=R^{\alpha}{ }_{\beta}-K K_{\beta}^{\alpha}-\frac{1}{6} \delta^{\alpha}{ }_{\beta}\left(R+2 \Lambda+K^{\mu}{ }_{\nu} K_{\mu}^{\nu}-K^{2}\right) \\
=R^{\alpha}{ }_{\beta}-K K^{\alpha}{ }_{\beta}-\frac{2}{3} \delta^{\alpha}{ }_{\beta} \Lambda, \\
\frac{\partial h_{\alpha \beta}}{\partial y}=2 h_{\alpha \gamma} K^{\gamma}{ }_{\beta},
\end{gathered}
$$

where $R_{\beta}^{\alpha}$ are the components of the Ricci tensor of the metric $h_{\alpha \beta}$ induced on the hypersurface $y=$ const, $R=R^{\alpha}{ }_{\alpha}$ is its scalar curvature, and $K=K^{\alpha}{ }_{\alpha}$ is the trace of the tensor of extrinsic curvature. The second equality in (11) is true by virtue of the 'constraint' equation (6). Equations (11) and (12) together with the 'constraint' equation (6) represent the $4+1$ splitting of the Einstein equations in Gaussian normal coordinates. The initial conditions for these equations are defined on the brane through Eq. (4).

\section{LINEAR PERTURBATIONS}

Linear perturbations of the RS1 model are well studied (see, e.g., $[19,20]$ and references therein). Here, we would like to see the modifications arising from the presence of the scalar-curvature terms in the action for the branes (nonzero values of the masses $m$ and

\footnotetext{
${ }^{2}$ It is interesting that, while the conformal anomaly term $R_{a b} R^{a b}-\frac{1}{3} R^{2}$ cannot be obtained by the variation of a local four-dimensional Lagrangian, the very same term is obtained via the variation of a local Lagrangian in the five-dimensional braneworld theory under investigation [14]. Also note that this term is absent in the original Randall-Sundrum model which has $m=0$.
} 
$\left.m_{*}\right)$. Several aspects of this setup were also studied in [8-11]. Our treatment in this and in the subsequent section is similar to that of [20].

The perturbed metric of our solution in Gaussian normal coordinates has the form (10) with

$$
h_{\alpha \beta}(x, y)=a^{2}(y)\left[\eta_{\alpha \beta}+\gamma_{\alpha \beta}(x, y)\right]
$$

where

$$
a(y)=\exp (-k y), \quad k=\frac{\sigma}{3 M^{3}},
$$

and we emphasize that $k$ can be positive as well as negative depending on the signs of $M$ and $\sigma$. The perturbations of the tensor of extrinsic curvature and of the Einstein tensor have the form

$$
\delta K_{\beta}^{\alpha}=\frac{1}{2} \frac{\partial \gamma_{\beta}^{\alpha}}{\partial y}, \quad G_{\alpha \beta}=-\frac{1}{2} \square \bar{\gamma}_{\alpha \beta}+\partial^{\gamma} \partial_{(\alpha} \bar{\gamma}_{\beta) \gamma}-\frac{1}{2} \eta_{\alpha \beta} \partial^{\gamma} \partial^{\delta} \bar{\gamma}_{\gamma \delta},
$$

where $\bar{\gamma}_{\alpha \beta}=\gamma_{\alpha \beta}-\frac{1}{2} \eta_{\alpha \beta} \gamma, \gamma=\gamma_{\alpha}^{\alpha}$, and $\square=\partial^{\alpha} \partial_{\alpha}$. Here and below, the indices of $\partial_{\alpha}$ and $\gamma_{\alpha \beta}$ are raised and lowered with respect to the flat metric $\eta_{\alpha \beta}$.

Using the freedom of choice of the coordinates $x^{\alpha}$ on the brane, one can choose the harmonic gauge in which $\partial^{\alpha} \bar{\gamma}_{\alpha \beta}=0$ on one of the branes. In this gauge, we have

$$
G_{\alpha \beta}=-\frac{1}{2} \square \bar{\gamma}_{\alpha \beta}
$$

on that brane.

In the unperturbed solution, the first (visible) brane is assumed to be at $y=0$, and the second (hidden) brane is at $y=\rho$. First, we consider the situation where the hidden brane does not have matter on it (stress-energy tensor equal to zero). Then, when studying perturbations, it is convenient to choose Gaussian normal coordinates with respect to the hidden brane. Thus, the hidden brane remains at $y=\rho$, while the position of the visible brane is linearly perturbed to become $y=\phi(x)$, which is the so-called radion degree of freedom. Let $m$ and $\sigma$ denote the Planck mass and tension of the visible brane, and let those of the hidden brane be $m_{*}$ and $\sigma_{*}=-\sigma$, respectively. The linearly perturbed boundary equation (4) on the second (hidden) brane becomes

$$
-m_{*}^{2} G_{\beta}^{\alpha}=M^{3} \delta S^{\alpha}{ }_{\beta},
$$

where $S^{\alpha}{ }_{\beta}=K^{\alpha}{ }_{\beta}-\delta^{\alpha}{ }_{\beta} K$, and we have taken into account that the extrinsic curvature is calculated with respect to the normal in the positive direction of $y$. Choosing harmonic coordinates on the hidden brane, for which (16) is satisfied, we have

$$
\frac{m_{*}^{2}}{a_{*}^{2}} \square \bar{\gamma}_{\beta}^{\alpha}=M^{3}\left(\frac{\partial \bar{\gamma}^{\alpha}{ }_{\beta}}{\partial y}+\frac{1}{2} \delta^{\alpha}{ }_{\beta} \frac{\partial \bar{\gamma}}{\partial y}\right),
$$

where $a_{*}=a(\rho)=e^{-k \rho}$ and $\bar{\gamma}=\bar{\gamma}_{\alpha}^{\alpha}$.

Linearization of the vacuum constraint equation (8) implies the condition $\square \bar{\gamma}=0$ on the hidden brane if 


$$
M^{6}+\frac{2}{3} \sigma_{*} m_{*}^{2} \neq 0
$$

which we assume to be the case. This condition and Eq. (18) implies the condition $\partial \bar{\gamma} / \partial y=0$ at the hidden brane. Then the Codazzi relation (9) implies the condition $\partial\left(\partial^{\alpha} \bar{\gamma}_{\alpha \beta}\right) / \partial y=0$ at the same brane.

Now we turn to Eqs. (11) and (12). Using (15), we can write the second-order differential equations for perturbations $\bar{\gamma}_{\alpha \beta}$ in the bulk. First, we verify that the Gaussian normal coordinates $x^{\alpha}$ remain harmonic in the bulk. We introduce the quantity

$$
v^{\alpha}=\partial_{\beta} \bar{\gamma}^{\beta \alpha}
$$

which is an indicator of the harmonicity of the coordinates $x^{\alpha}$ on the hypersurface $y=$ const. Then we can write the system of differential equations for $v^{\alpha}$ and $\bar{\gamma}$ that stems from system (11), (12):

$$
\frac{\partial^{2} v^{\alpha}}{\partial y^{2}}=4 k \frac{\partial v^{\alpha}}{\partial y}+k \frac{\partial\left(\partial^{\alpha} \bar{\gamma}\right)}{\partial y}, \quad \frac{\partial^{2} \bar{\gamma}}{\partial y^{2}}=-\frac{1}{a^{2}}\left(2 \partial_{\alpha} v^{\alpha}+\square \bar{\gamma}\right)+8 k \frac{\partial \bar{\gamma}}{\partial y},
$$

with the following boundary conditions at the hidden brane $(y=\rho)$ :

$$
v^{\alpha}=0, \quad \frac{\partial v^{\alpha}}{\partial y}=0, \quad \square \bar{\gamma}=0, \quad \frac{\partial \bar{\gamma}}{\partial y}=0 .
$$

The unique solution of system (21) in the bulk with the boundary conditions (22) is

$$
v^{\alpha}(x, y) \equiv 0, \quad \bar{\gamma}(x, y) \equiv \bar{\gamma}(x), \quad \square \bar{\gamma}(x)=0
$$

In particular, this means that the Gaussian normal coordinates which we are using remain harmonic with respect to $x$ all over the bulk.

Taking into account relations (23), from (11), (12) one obtains the system of equations for perturbations in the bulk:

$$
\frac{\partial^{2} \bar{\gamma}_{\alpha \beta}}{\partial y^{2}}-4 k \frac{\partial \bar{\gamma}_{\alpha \beta}}{\partial y}+\frac{1}{a^{2}} \square \bar{\gamma}_{\alpha \beta}=0
$$

with the boundary condition at $y=\rho$ which stems from (18):

$$
\frac{\partial \bar{\gamma}_{\alpha \beta}}{\partial y}=\frac{m_{*}^{2}}{M^{3} a_{*}^{2}} \square \bar{\gamma}_{\alpha \beta} .
$$

To obtain the boundary equations on the visible brane, one must take into account its 'bending' in the bulk: $y=\phi(x)$. The induced metric on the visible brane in the linear approximation becomes

$$
h_{\alpha \beta}^{\mathrm{vis}}=(1-2 k \phi) \eta_{\alpha \beta}+\gamma_{\alpha \beta}
$$

so that its perturbation is

$$
\gamma_{\alpha \beta}^{\mathrm{vis}}=\gamma_{\alpha \beta}-2 k \phi \eta_{\alpha \beta}, \quad \bar{\gamma}_{\alpha \beta}^{\mathrm{vis}}=\bar{\gamma}_{\alpha \beta}+2 k \phi \eta_{\alpha \beta}
$$


Substituting it to the boundary condition at the visible brane

$$
m^{2} G_{\beta}^{\alpha}=M^{3} \delta S_{\beta}^{\alpha}+\tau_{\beta}^{\alpha},
$$

we obtain the boundary condition at $y=0$ :

$$
m^{2} G_{\alpha \beta} \equiv-\frac{m^{2}}{2} \square \bar{\gamma}_{\alpha \beta}+2 m^{2} k\left(\partial_{\alpha} \partial_{\beta}-\eta_{\alpha \beta} \square\right) \phi=\tau_{\alpha \beta}+M^{3}\left(\eta_{\alpha \beta} \square-\partial_{\alpha} \partial_{\beta}\right) \phi+\frac{1}{2} M^{3} \frac{\partial \bar{\gamma}_{\alpha \beta}}{\partial y} .
$$

Taking trace of this equation, we obtain the equation for the radion field $\phi$ :

$$
-3 A \square \phi=\tau,
$$

where $\tau \equiv \eta^{\alpha \beta} \tau_{\alpha \beta}$ is the trace of the stress-energy tensor, and $A=M^{3}+2 \mathrm{~km}^{2}$. Thus, the radion field is coupled to the trace of the stress-energy tensor, as is the case in the Randall-Sundrum model [19], but with different coupling constant. Using Eq. (30), from (29) we obtain

$$
-\frac{m^{2}}{2} \square \bar{\gamma}_{\alpha \beta}=\tau_{\alpha \beta}-\frac{1}{3} \eta_{\alpha \beta} \tau-A \partial_{\alpha} \partial_{\beta} \phi+\frac{1}{2} M^{3} \frac{\partial \bar{\gamma}_{\alpha \beta}}{\partial y}
$$

Now we have to solve the bulk equations (24) with the boundary conditions (30), (25) and (31). Proceeding to the Fourier transform with momenta $p_{\alpha}$ in the coordinates $x^{\alpha}$ and omitting the tensor indices, we have for the Fourier image $\psi(q, y)$ of $\bar{\gamma}_{\alpha \beta}(x, y)$ :

$$
\psi^{\prime \prime}-4 k \psi^{\prime}+q^{2} e^{2 k y} \psi=0
$$

where the prime denotes the derivative with respect to $y$, and $q=\sqrt{-p^{2}}$ (here we assume $p^{2} \equiv p^{\alpha} p_{\alpha} \leq 0$; the tachyonic case will be studied in Sec. V). After the standard change of variable and function

$$
z(y)=\frac{q e^{k y}}{k}, \quad \psi(z)=z^{2} \chi(z)
$$

we get the equation

$$
z^{2} \chi^{\prime \prime}+z \chi^{\prime}+\left(z^{2}-4\right) \chi=0
$$

in which the prime denotes the derivative with respect to $z$. Note that $z(y)$ is a monotonic function of $y$ for both signs of $k$, but the sign of $z$ coincides with the sign of the constant $k$. The boundary conditions follow from (31) and (25):

$$
\begin{aligned}
-m^{2} k^{2} z_{0}^{2} \chi\left(z_{0}\right) & =\frac{2 T}{z_{0}^{2}}+k M^{3}\left[z_{0} \chi^{\prime}\left(z_{0}\right)+2 \chi\left(z_{0}\right)\right], \quad z_{0}=z(0)=\frac{q}{k} \\
m_{*}^{2} k^{2} z_{*}^{2} \chi\left(z_{*}\right) & =k M^{3}\left[z_{*} \chi^{\prime}\left(z_{*}\right)+2 \chi\left(z_{*}\right)\right], \quad z_{*}=z(\rho)=\frac{q}{k} e^{k \rho}
\end{aligned}
$$

where $T$ stands for the Fourier transform of the expression 


$$
T_{\alpha \beta}=\tau_{\alpha \beta}-\frac{1}{3} \eta_{\alpha \beta} \tau-A \partial_{\alpha} \partial_{\beta} \phi
$$

with tensor indices omitted.

The general solution of the Bessel equation (34) is given by

$$
\chi(z)=P J_{2}(|z|)+Q Y_{2}(|z|),
$$

where $J_{2}$ and $Y_{2}$ are the Bessel functions, $P$ and $Q$ are constants, and the modulus of $z$ reflects the fact that the domain of $z$ is positive or negative depending on the sign of $k$.

Using the recurrence relations

$$
z J_{2}^{\prime}(z)+2 J_{2}(z)=z J_{1}(z), \quad z Y_{2}^{\prime}(z)+2 Y_{2}(z)=z Y_{1}(z),
$$

we obtain from (35) and (36):

$$
\begin{aligned}
-m^{2} k^{2} z_{0}^{2}\left[P J_{2}\left(\left|z_{0}\right|\right)+Q Y_{2}\left(\left|z_{0}\right|\right)\right] & =\frac{2 T}{z_{0}^{2}}+k M^{3}\left|z_{0}\right|\left[P J_{1}\left(\left|z_{0}\right|\right)+Q Y_{1}\left(\left|z_{0}\right|\right)\right], \\
m_{*}^{2} k^{2} z_{*}^{2}\left[P J_{2}\left(\left|z_{*}\right|\right)+Q Y_{2}\left(\left|z_{*}\right|\right)\right] & =k M^{3}\left|z_{*}\right|\left[P J_{1}\left(\left|z_{*}\right|\right)+Q Y_{1}\left(\left|z_{*}\right|\right)\right],
\end{aligned}
$$

solving which, one finds the constants $P$ and $Q$ and obtains the solution for $\psi(z)$ :

$$
\psi(z)=\left(\frac{6 T z^{2}}{\sigma\left|z_{0}\right|^{3}}\right) \frac{C_{Y}^{*} J_{2}(|z|)-C_{J}^{*} Y_{2}(|z|)}{C_{Y}^{0} C_{J}^{*}-C_{J}^{0} C_{Y}^{*}},
$$

where the constants are given by

$$
\begin{array}{ll}
C_{Y}^{0}=Y_{1}\left(\left|z_{0}\right|\right)+\frac{m^{2}}{M^{3}} k\left|z_{0}\right| Y_{2}\left(\left|z_{0}\right|\right), & C_{J}^{0}=J_{1}\left(\left|z_{0}\right|\right)+\frac{m^{2}}{M^{3}} k\left|z_{0}\right| J_{2}\left(\left|z_{0}\right|\right), \\
C_{Y}^{*}=Y_{1}\left(\left|z_{*}\right|\right)-\frac{m_{*}^{2}}{M^{3}} k\left|z_{*}\right| Y_{2}\left(\left|z_{*}\right|\right), & C_{J}^{*}=J_{1}\left(\left|z_{*}\right|\right)-\frac{m_{*}^{2}}{M^{3}} k\left|z_{*}\right| J_{2}\left(\left|z_{*}\right|\right) .
\end{array}
$$

These results differ from the similar results $[19,20]$ of the RS1 model by the presence of the terms containing the brane Planck masses $m$ and $m_{*}$ in Eqs. (43).

The spectrum of the model is determined by the equality of the denominator of (42) to zero. Introducing the dimensionless variable $s=q /|k|$ and parameters $\mu=\mathrm{km}^{2} / \mathrm{M}^{3}$, $\mu_{*}=k m_{*}^{2} / M^{3}$, and $\alpha=e^{k \rho}$, we obtain the following equation for the spectrum:

$$
F_{1}(s)+\mu s F(s)+\alpha \mu_{*} s F_{*}(s)+\alpha \mu \mu_{*} s^{2} F_{2}(s)=0,
$$

where

$$
\begin{aligned}
& F_{1}(s)=J_{1}(s) Y_{1}(\alpha s)-J_{1}(\alpha s) Y_{1}(s) \\
& F(s)=J_{2}(s) Y_{1}(\alpha s)-J_{1}(\alpha s) Y_{2}(s) \\
& F_{*}(s)=J_{2}(\alpha s) Y_{1}(s)-J_{1}(s) Y_{2}(\alpha s) \\
& F_{2}(s)=J_{2}(\alpha s) Y_{2}(s)-J_{2}(s) Y_{2}(\alpha s) .
\end{aligned}
$$

If both masses $m$ and $m_{*}$ are nonzero, then the ultraviolet asymptotics of the spectrum for the Kaluza-Klein modes is determined by the zeros of the last term in (44), so that 


$$
s_{n} \sim \frac{\pi n}{\alpha-1}, \quad n \gg 1
$$

which coincides with the asymptotics of the spectrum in the Randall-Sundrum model, determined by the zeros of the first term in (44).

If $m \neq 0, m_{*}=0$, then the asymptotics of the spectrum is determined by the second term in (44):

$$
s_{n} \sim \frac{\pi n-\frac{\pi}{2}}{\alpha-1}, \quad n \gg 1
$$

If $m=0, m_{*} \neq 0$, then it is determined by the third term in (44):

$$
s_{n} \sim \frac{\pi n+\frac{\pi}{2}}{\alpha-1}, \quad n \gg 1
$$

In these last two cases, the spectrum is somewhat shifted.

\section{LINEARIZED EQUATIONS IN THE ZERO-MODE APPROXIMATION}

In the zero-mode approximation [19,20], one considers the limit as $q \rightarrow 0$. In this limit, using Eqs. (29), (30), and (42) and expanding all functions of $q=\sqrt{-p^{2}}$ in powers of $q$, we obtain the linearized gravity equation in the case of matter present only on the visible brane:

$$
G_{\alpha \beta}=\frac{2 k}{A-B e^{-2 k \rho}}\left[\tau_{\alpha \beta}-\frac{B e^{-2 k \rho}}{3 A}\left(\eta_{\alpha \beta}-\frac{p_{\alpha} p_{\beta}}{p^{2}}\right) \tau\right]+\mathcal{O}\left(p^{2}\right)
$$

where it should be stressed that $G_{\alpha \beta}$ is the Einstein tensor of the induced metric (26) on the brane, and the constants $A$ and $B$ are given by ${ }^{3}$

$$
A=M^{3}+2 k m^{2}, \quad B=M^{3}-2 k m_{*}^{2} .
$$

The effective Newton's constant $G_{\mathrm{N}}$ is given by the relation

$$
8 \pi G_{\mathrm{N}}=\frac{2 k}{A-B e^{-2 k \rho}}
$$

and one should note the extra contribution from the radion in (49), which involves the trace of the stress-energy tensor. ${ }^{4}$ If $k>0$, this contribution is exponentially suppressed for large separations between the branes, $k \rho \gg 1$.

\footnotetext{
${ }^{3}$ The constant $A$ is the same as in Eq. (30).

${ }^{4}$ In Eq. (49) as well as in similar equations of this section, we formally express the radion field through the trace of the stress-energy tensor using Eq. (30), similarly to how it is done, e.g., in $[20]$.
} 
If matter is present only on the hidden brane, then it still induces curvature on the visible brane $[19,20]$. In our theory, we obtain the result

$$
G_{\alpha \beta}=\frac{2 k}{A e^{2 k \rho}-B}\left[\tau_{\alpha \beta}^{*}-\frac{e^{-2 k \rho}}{3}\left(\eta_{\alpha \beta}-\frac{p_{\alpha} p_{\beta}}{p^{2}}\right) \tau^{*}\right]+\mathcal{O}\left(p^{2}\right),
$$

where $\tau_{\alpha \beta}^{*}$ is the stress-energy tensor on the hidden brane, and $\tau^{*}$ is its trace. If both branes contain matter, then the results on the right-hand sides of (49) and (52) simply add together.

A few comments are in order about the obtained results. First of all, in the limit of zero Planck masses for the branes, $m=m_{*}=0$, we have $A=B=M^{3}$, and they turn to the results previously obtained for the Randall-Sundrum two-brane model $[19,20]$. The presence of two new mass parameters $m$ and $m_{*}$ extends the freedom of the model. Thus, if the constant $B$ turns out to be sufficiently small, then the scalar contribution to the right-hand side of (49), proportional to the trace of the stress-energy tensor, may become negligibly small. Note, however, that it is not possible to set either the constant $A$ or the constant $B$ exactly to zero in our expressions since, in this case, the theory becomes singular. This can be seen, e.g., from Eq. (8), in which the first (linear in curvature) term is proportional to $A$ [the same property is observed in the general equation (7)]. In particular, the nonzero value of the constant $B$ was already assumed in the linearization scheme [see Eq. (19)]. The special cases where either $A$ or $B$ is equal to zero must be studied separately. Some results in this direction were recently reported in [10], where it was pointed out that the linearized theory possesses some additional symmetry in this case.

To see how this degeneracy arises in some more detail, we turn to the Gauss identity (5) again and, following the procedure first employed in [21], contract it once on the brane using equations (3) and (4). We obtain the effective equation on the brane that generalizes the result of [21] to the presence of the brane curvature term:

$$
G_{a b}+\Lambda_{\mathrm{RS}} \frac{M^{3}}{A} h_{a b}=\frac{2 \sigma}{3 M^{3} A} \tau_{a b}+\frac{1}{M^{3} A} Q_{a b}-\frac{M^{3}}{A} W_{a b},
$$

where $\Lambda_{\mathrm{RS}}$ is given by (2),

$$
Q_{a b}=\frac{1}{3} E E_{a b}-E_{a c} E_{b}^{c}+\frac{1}{2}\left(E_{c d} E^{c d}-\frac{1}{3} E^{2}\right) h_{a b}
$$

is the quadratic expression with respect to the 'bare' Einstein equation $E_{a b} \equiv m^{2} G_{a b}-\tau_{a b}$ on the brane, $E=h^{a b} E_{a b}$, and $W_{a b} \equiv h_{a}^{c} h_{b}^{e} W_{c d e f} n^{d} n^{f}$ is a projection of the bulk Weyl tensor $W_{a b c d}$ to the brane. One can see that all the couplings in (53), including the effective cosmological and gravitational constants, are inversely proportional to the constant $A$, which indicates that the theory becomes degenerate in the case $A=0$. In the absence of the curvature term on the brane $(m=0)$, we have $A=M^{3}$, which brings us to the original result of $[21]$.

Our second remark is that, unlike in the original Randall-Sundrum model $\left(m=m_{*}=0\right)$, in our theory the sign of the constant $M$ is not fixed by the zero-mode approximation: apart from the scalar contribution described by the trace of the stress-energy tensor, matching with the general-relativity limit fixes only the sign of the overall constant in (49) and (52). In particular, for a sufficiently small absolute value of $M$, namely, $\left|M^{3} / 2 k\right| \ll m^{2}, m_{*}^{2}$, the 
sign of $M$ does not matter. In the formal limit $M \rightarrow 0$ with $k>0$ (hence, $k \rightarrow+\infty$ ), the equation for the visible brane (49) turns to the usual linearized Einstein equation. In the formal simultaneous limit $M \rightarrow 0$ and $\sigma \rightarrow 0$ so that $k=\sigma / M^{3}$ is fixed, expressions (49) and (52) become

$$
G_{\alpha \beta}=\frac{1}{m^{2}+m_{*}^{2} e^{-2 k \rho}}\left[\tau_{\alpha \beta}+\frac{m_{*}^{2} e^{-2 k \rho}}{3 m^{2}}\left(\eta_{\alpha \beta}-\frac{p_{\alpha} p_{\beta}}{p^{2}}\right) \tau\right]+\mathcal{O}\left(p^{2}\right)
$$

and

$$
G_{\alpha \beta}=\frac{1}{m^{2} e^{2 k \rho}+m_{*}^{2}}\left[\tau_{\alpha \beta}^{*}-\frac{e^{-2 k \rho}}{3}\left(\eta_{\alpha \beta}-\frac{p_{\alpha} p_{\beta}}{p^{2}}\right) \tau^{*}\right]+\mathcal{O}\left(p^{2}\right),
$$

respectively. However, in the following section we will see that the massive gravitational modes in the theory with negative value of $M$ have ghost-like nature.

Finally, we note that our result does not explicitly contain the constant $\sigma$ but contains it only in the combination $k=\sigma / 3 M^{3}$. Therefore, for one and the same effective law of gravity (49) and (52), the visible brane can have either positive or negative brane tension, depending on the sign of $M$. In particular, the zero-mode graviton is 'localized' around the visible brane $(k>0)$ even if its tension is negative, provided $M$ is also negative. If $k>0$, then, in the limit $\rho \rightarrow \infty$, we pass to the one-brane model in Eq. (49), which has the form of the corresponding equation of general relativity.

\section{TACHYONIC MODES AND GHOSTS}

In the original Randall-Sundrum model, negative values of the bulk Planck mass $M$ are nonphysical because this leads to negative effective Newton's constant. This can be seen by setting $m=m_{*}=0$ in Eq. (51), thus having $A=B=M^{3}$ in it. If $M<0$, then the effective Newton's constant is negative for any sign of $k$, which means that the massless graviton becomes a ghost.

The presence of the curvature terms in the action for the brane relaxes the situation with the massless gravitational modes and thus relaxes the necessity of dealing only with positive values of $M$. Negative values of $M$ are of interest in view of some of the braneworld cosmological models with negative brane tension, in particular, the model of disappearing dark energy $[14,15]$, as discussed in the introduction. However, unlike in the pure RandallSundrum case, the presence of the curvature terms in the action for the branes leads to a possibility of unwanted tachyonic modes and ghost-like character of the massive modes in the gravitational sector of the theory if $M<0$. In this section, we demonstrate that there can be only one or two tachyonic mass eigenvalues in the theory under consideration and determine the range of parameters for which tachyonic modes do not exist. We also show that the massive gravitational modes have ghost-like character in the case of negative $M$.

In looking for tachyonic modes, one needs to solve Eq. (32) for $q^{2}=-p^{2}<0$, i.e.,

$$
\psi^{\prime \prime}-4 k \psi^{\prime}-p^{2} e^{2 k y} \psi=0, \quad p^{2}>0,
$$

where the prime denotes the derivative with respect to $y$. After the standard change of variable and function 


$$
z(y)=p e^{k y} / k, \quad \psi(z)=z^{2} \chi(z)
$$

we get the equation for the new function $\chi(z)$ :

$$
z^{2} \chi^{\prime \prime}+z \chi^{\prime}-\left(z^{2}+4\right) \chi=0
$$

in which the prime denotes the derivative with respect to $z$. With matter present on the visible brane only, the boundary conditions are similar to (35) and (36):

$$
\begin{aligned}
m^{2} k^{2} z_{0}^{2} \chi\left(z_{0}\right) & =\frac{2 T}{z_{0}^{2}}+k M^{3}\left[z_{0} \chi^{\prime}\left(z_{0}\right)+2 \chi\left(z_{0}\right)\right], \quad z_{0}=z(0)=\frac{p}{k} \\
-m_{*}^{2} k^{2} z_{*}^{2} \chi\left(z_{*}\right) & =k M^{3}\left[z_{*} \chi^{\prime}\left(z_{*}\right)+2 \chi\left(z_{*}\right)\right], \quad z_{*}=z(\rho)=\frac{p}{k} e^{k \rho}
\end{aligned}
$$

where $T$ is the Fourier transform of expression (37), with tensor indices omitted.

Solution of (59) is now given by the modified Bessel functions $I_{2}$ and $K_{2}$ :

$$
\chi(z)=P I_{2}(|z|)+Q K_{2}(|z|),
$$

where $P$ and $Q$ are constants, and the modulus of $z$ again reflects the fact that the domain of $z$ is positive or negative depending on the sign of $k$. The recurrence relations of type (39) are valid also for the modified Bessel functions:

$$
z I_{2}^{\prime}(z)+2 I_{2}(z)=z I_{1}(z), \quad z K_{2}^{\prime}(z)+2 K_{2}(z)=-z K_{1}(z)
$$

and we can use them in deriving the solution similar to (42):

$$
\psi(z)=\left(\frac{6 T z^{2}}{\sigma\left|z_{0}\right|^{3}}\right) \frac{C_{K}^{*} I_{2}(|z|)+C_{I}^{*} K_{2}(|z|)}{C_{I}^{*} C_{K}^{0}-C_{I}^{0} C_{K}^{*}}
$$

where

$$
\begin{array}{ll}
C_{I}^{0}=I_{1}\left(\left|z_{0}\right|\right)-\frac{m^{2}}{M^{3}} k\left|z_{0}\right| I_{2}\left(\left|z_{0}\right|\right), & C_{K}^{0}=K_{1}\left(\left|z_{0}\right|\right)+\frac{m^{2}}{M^{3}} k\left|z_{0}\right| K_{2}\left(\left|z_{0}\right|\right) \\
C_{I}^{*}=I_{1}\left(\left|z_{*}\right|\right)+\frac{m_{*}^{2}}{M^{3}} k\left|z_{*}\right| I_{2}\left(\left|z_{*}\right|\right), & C_{K}^{*}=K_{1}\left(\left|z_{*}\right|\right)-\frac{m_{*}^{2}}{M^{3}} k\left|z_{*}\right| K_{2}\left(\left|z_{*}\right|\right) .
\end{array}
$$

At this point, we note that the restriction to the brane at $y=0$ and the limit of brane separation $\rho \rightarrow \infty$ brings expression (64) to the form obtained for the one-brane case in [13]. Our result generalizes it to the case of two branes with arbitrary sign of the bulk gravitational constant.

Tachyonic modes correspond to those values of $p$ for which the denominator of (64) turns to zero:

$$
C_{I}^{*} C_{K}^{0}-C_{I}^{0} C_{K}^{*}=0
$$

Since the transformation $p \rightarrow e^{-k \rho} p$ followed by $k \rightarrow-k$ and $m \leftrightarrow m_{*}$ does not change the spectrum of the theory, it is sufficient to study only the case $k>0$. Using the dimensionless 
variable $s=p / k$ and parameters $\mu=k m^{2} / M^{3}, \mu_{*}=k m_{*}^{2} / M^{3}$, and $\alpha=e^{k \rho}$, we obtain the equation for tachyonic modes:

$$
E(s) \equiv D_{1}(s)+\mu s D(s)+\alpha \mu_{*} s D_{*}(s)+\alpha \mu \mu_{*} s^{2} D_{2}(s)=0,
$$

where

$$
\begin{aligned}
& D_{1}(s)=I_{1}(\alpha s) K_{1}(s)-I_{1}(s) K_{1}(\alpha s), \\
& D(s)=I_{2}(s) K_{1}(\alpha s)+I_{1}(\alpha s) K_{2}(s), \\
& D_{*}(s)=I_{1}(s) K_{2}(\alpha s)+I_{2}(\alpha s) K_{1}(s), \\
& D_{2}(s)=I_{2}(\alpha s) K_{2}(s)-I_{2}(s) K_{2}(\alpha s) .
\end{aligned}
$$

Since $\alpha>1$ for $k>0$, all functions in (68) are strictly positive for positive $s$. This implies that tachyonic modes are absent in the case $\mu, \mu_{*}>0$, or, equivalently, $M>0$.

However, tachyonic modes may be present in the opposite case $M<0$. It is possible to indicate the corresponding range of parameters where tachyonic modes are present or absent. To do this, it is convenient to introduce the following auxiliary function of two variables $s$ and $\bar{s}$ :

$$
\bar{E}(s, \bar{s}) \equiv D_{1}(s)+\mu \bar{s} D(s)+\alpha \mu_{*} \bar{s} D_{*}(s)+\alpha \mu \mu_{*} \bar{s}^{2} D_{2}(s)
$$

[to be compared with (67)]. By construction, $\bar{E}(s, s) \equiv E(s)$.

First, we consider the case where both $\mu$ and $\mu_{*}$ are nonzero (in the present case, they are then both negative). Then the equation

$$
\bar{E}(s, \bar{s})=0
$$

gives the two branches of solutions with respect to $\bar{s}$ :

$$
\bar{s}_{ \pm}(s)=\frac{\left|\mu D(s)+\alpha \mu_{*} D_{*}(s)\right| \pm \sqrt{\left(\mu D(s)+\alpha \mu_{*} D_{*}(s)\right)^{2}-4 \alpha \mu \mu_{*} D_{1}(s) D_{2}(s)}}{2 \alpha \mu \mu_{*} D_{2}(s)}
$$

and solving the original equation (67) is equivalent to solving one of the equations

$$
\bar{s}_{ \pm}(s)=s .
$$

It is easy to verify that the expression under the square root of (71) is strictly positive for positive $s$ so that the two solutions $\bar{s}_{ \pm}(s)$ exist for all $s>0$ and are positive. The asymptotic behavior of these solutions for small and large $s$ can easily be found:

$$
\begin{gathered}
\bar{s}_{+}(s) \sim \frac{4\left|\alpha^{2} \mu+\mu_{*}\right|}{\left(\alpha^{4}-1\right) \mu \mu_{*} s}, \quad \bar{s}_{-}(s) \sim \frac{\left(\alpha^{2}-1\right) s}{2\left|\alpha^{2} \mu+\mu_{*}\right|}, \quad s \rightarrow 0, \\
\bar{s}_{ \pm}(s) \rightarrow \frac{\left|\mu+\alpha \mu_{*}\right| \pm\left|\mu-\alpha \mu_{*}\right|}{2 \alpha \mu \mu_{*}}=\mathrm{const}, \quad s \rightarrow \infty .
\end{gathered}
$$


From these expressions it is clear that the graph of $\bar{s}_{+}(s)$ crosses the graph of $f(s)=s$ at least once for any values of parameters in the range $\mu, \mu_{*}<0$ under consideration. Thus, Eq. (72) has a solution, and at least one tachyonic mass eigenvalue is present in this range of parameters. Numerical computation indicates that there is exactly one solution connected with the branch $\bar{s}_{+}(s)$ in this case.

It is also clear that the graph of $\bar{s}_{-}(s)$ definitely crosses the graph of $f(s)=s$ in the case where $\bar{s}_{-}^{\prime}(0)>1$, or

$$
\frac{\left(\alpha^{2}-1\right)}{2\left|\alpha^{2} \mu+\mu_{*}\right|}>1
$$

Again, numerical computation indicates that there is only one tachyonic solution connected with the branch $\bar{s}_{-}(s)$ in this case. They also indicate that tachyonic modes connected with the branch $\bar{s}_{-}(s)$ are absent in the case of the opposite inequality in (75).

Thus, tachyonic modes exist for all values of parameters in the range $\mu, \mu_{*}<0$. In the case under consideration, $M<0$, one can expect tachyonic modes to be absent only if one of the brane Planck masses $m$ or $m_{*}$ is zero. ${ }^{5}$ We show that this is indeed the case and determine the range of masses and brane separations for which tachyonic modes are absent.

In the case $\mu<0, \mu_{*}=0$, the function $\bar{E}(s, \bar{s})$ given by Eq. (69) takes the simple form

$$
\bar{E}(s, \bar{s}) \equiv D_{1}(s)+\mu \bar{s} D(s),
$$

and Eq. (70) has a single solution with respect to $\bar{s}$ :

$$
\bar{s}(s)=-\frac{D_{1}(s)}{\mu D(s)}=\frac{D_{1}(s)}{|\mu| D(s)} .
$$

It can be verified that the function $D_{1}(s) / D(s)$ is convex upwards, so that the equation $\bar{s}(s)=s$ has exactly one solution or no solutions in the range $s>0$ depending on the value of the derivative $\bar{s}^{\prime}(0)$. Specifically, a solution exists if $\bar{s}^{\prime}(0)>1$, and there are no solutions in the opposite case $\bar{s}^{\prime}(0) \leq 1$. Calculating the derivative $\bar{s}^{\prime}(0)$, we obtain that exactly one tachyonic mass eigenvalue is present in the theory if

$$
|\mu|<\frac{1}{2}\left(1-\alpha^{-2}\right)
$$

and tachyonic modes are absent if the value of the Planck mass $m$ is sufficiently large, namely, if

$$
|\mu| \geq \frac{1}{2}\left(1-\alpha^{-2}\right)
$$

In the limit of infinite separation between branes, $\alpha=e^{k \rho} \rightarrow \infty$, the condition of absence of tachyonic modes becomes $|\mu| \geq 1 / 2$, which coincides with the condition $A \geq 0$, where $A$ is

\footnotetext{
${ }^{5}$ Tachyonic modes are obviously absent in the Randall-Sundrum model $\left(m=m_{*}=0\right)$ even in the case of negative bulk gravitational constant $(M<0)$, but this model is already excluded as resulting in negative effective Newton's constant on the brane (see the beginning of this section).
} 
given by (50). Interestingly, this is also the condition of positivity of the effective Newton's constant in the zero-mode approximation (49), (52) in the same limit.

The case $\mu=0, \mu_{*}<0$ is analyzed in quite a similar way. Now the function $\bar{E}(s, \bar{s})$ given by Eq. (69) takes the form

$$
\bar{E}(s, \bar{s}) \equiv D_{1}(s)+\alpha \mu_{*} \bar{s} D_{*}(s),
$$

and Eq. (70) has one solution with respect to $\bar{s}$ :

$$
\bar{s}(s)=-\frac{D_{1}(s)}{\alpha \mu_{*} D_{*}(s)}=\frac{D_{1}(s)}{\alpha\left|\mu_{*}\right| D_{*}(s)} .
$$

Again, it can be verified that the equation $\bar{s}(s)=s$ has exactly one solution in the range $s>0$ if $\bar{s}^{\prime}(0)>1$, and there are no solutions in the opposite case $\bar{s}^{\prime}(0) \leq 1$. Calculating the derivative $\bar{s}^{\prime}(0)$, we obtain that exactly one tachyonic mass eigenvalue is present in the theory if

$$
\left|\mu_{*}\right|<\frac{1}{2}\left(\alpha^{2}-1\right),
$$

and tachyonic modes are absent if the value of the Planck mass $m_{*}$ is sufficiently large, namely, if

$$
\left|\mu_{*}\right| \geq \frac{1}{2}\left(\alpha^{2}-1\right) .
$$

We note that our method of finding the range of parameters where tachyonic terms are present or absent is not restricted to the model under investigation and can be used whenever the equation for tachyonic modes has the form (67), as is the case, e.g., in the theory with arbitrary signs of the brane gravitational constants [8-11] and/or with the Gauss-Bonnet action in the bulk [8].

The issue of ghosts in the gravitational sector of the complementary theory with positive value of $M$ but arbitrary signs of the brane gravitational constants was considered in [9], and we apply the results obtained therein to our case. First, we start with the radion. The radion degree of freedom in our formalism is connected with the possibility of brane bending in the bulk. After identifying the physical degrees of freedom for the radion, one can obtain the conditions for its ghost-free character in our model using the results of [9]:

$$
M^{3}\left(\frac{1}{1-2 \mu_{*}}-\frac{e^{-2 k \rho}}{1+2 \mu}\right) \geq 0
$$

which we expressed in terms of our parameters $\mu=k m^{2} / M^{3}$ and $\mu_{*}=k m_{*}^{2} / M^{3}$ restricting ourselves to the case $k>0$ and taking into account that $M$ can be of any sign. Then the conditions of absence of both tachyons and radion ghosts in the case $M<0$ following from (79), (83), and (84) are

$$
\mu_{*}=0, \quad \frac{1}{2}\left(1-e^{-2 k \rho}\right) \leq|\mu|<\frac{1}{2}, \quad \text { and } \quad \mu=0, \quad\left|\mu_{*}\right| \geq \frac{1}{2}\left(e^{2 k \rho}-1\right) .
$$

These conditions on the constants of the theory can be seen to be rather restrictive. 
Following [9], we can also show that the massive gravitational modes in the theory under consideration have ghost-like nature. For free metric perturbations in the form (13), (14) described by the transverse traceless modes $\gamma_{\alpha \beta}(x, y)$ with the boundary conditions (25) and (31) in which we set $\phi=0$ and $\tau_{\alpha \beta}=0$, one obtains the gravitational part of action (1) to quadratic order in the form

$$
\begin{aligned}
S & =\frac{M^{3}}{2} \int_{0}^{\rho} d y e^{-2 k y} \int d x\left(\gamma^{\alpha \beta} \square \gamma_{\alpha \beta}-e^{-2 k y} \partial_{y} \gamma^{\alpha \beta} \partial_{y} \gamma_{\alpha \beta}\right) \\
& +\frac{m^{2}}{2} \int_{y=0} d x \gamma^{\alpha \beta} \square \gamma_{\alpha \beta}+\frac{m_{*}^{2}}{2} e^{-2 k \rho} \int_{y=\rho} d x \gamma^{\alpha \beta} \square \gamma_{\alpha \beta} .
\end{aligned}
$$

Expanding the perturbation in the modes $\psi(q, y)$ that are solutions of Eq. (32) with the corresponding boundary conditions,

$$
\gamma_{\alpha \beta}(x, y)=\sum_{q} \chi_{\alpha \beta}(q, x) \psi(q, y),
$$

substituting this expansion into action (86), and using the orthogonality condition

$$
M^{3} \int_{0}^{\rho} d y e^{-2 k y} \psi\left(q_{1}, y\right) \psi\left(q_{2}, y\right)+m^{2} \psi\left(q_{1}, 0\right) \psi\left(q_{2}, 0\right)+m_{*}^{2} e^{-2 k \rho} \psi\left(q_{1}, \rho\right) \psi\left(q_{2}, \rho\right)=0
$$

for $q_{1} \neq q_{2}$, we arrive at the following quadratic effective action (cf. with [9]):

$$
S=\frac{1}{2} \sum_{q} C_{q} \int d x \chi^{\alpha \beta}(q, x)\left(\square-q^{2}\right) \chi_{\alpha \beta}(q, x),
$$

where

$$
C_{q}=M^{3} \int_{0}^{\rho} d y e^{-2 k y}[\psi(q, y)]^{2}+m^{2}[\psi(q, 0)]^{2}+m_{*}^{2} e^{-2 k \rho}[\psi(q, \rho)]^{2} .
$$

For the massless mode $(q=0)$, we have $\psi(0, y) \equiv$ const, and the constant $C_{0}$ is given by

$$
C_{0}=\frac{M^{3}}{2 k}[\psi(0,0)]^{2}\left[1+2 \mu-\left(1-2 \mu_{*}\right) e^{-2 k \rho}\right]
$$

and is positive in all cases in which tachyonic modes are absent in the theory, as can be seen from conditions (79) and (83). Thus, the massless graviton is not a ghost. However, for the massive modes $(q \neq 0)$, using Eq. (32), one can obtain the expression

$$
C_{q}=\frac{M^{3}}{q^{2}} \int_{0}^{\rho} d y e^{-4 k y}\left[\psi^{\prime}(q, y)\right]^{2}, \quad q \neq 0,
$$

which shows that the massive modes have positive norm in the case $M>0$, and have ghost-like nature in the case $M<0$.

\section{CORRECTIONS TO NEWTON'S LAW}

In this section, we compute the gravitational potential $V(r)$ on the visible brane induced by a static point source located on the visible or hidden brane and determine corrections to the Newton's law in the physically reasonable case $k>0$, i.e., where the zero-mode graviton is localized around the visible brane. Our starting formula is

$$
h_{00}^{(\mathrm{vis})}=-(1+2 V),
$$

where $h_{\alpha \beta}^{(\mathrm{vis})}$ is the induced metric on the visible brane. 


\section{A. Matter source on the visible brane}

If matter source is on the visible brane only, then the induced metric $h_{\alpha \beta}^{\text {(vis) }}$ is given by Eq. (26). The stress-energy tensor of a static point source of mass $\mathcal{M}$ is $\tau_{00}=\mathcal{M} \delta(\vec{r})$ with other components being zero, and $\tau=-\tau_{00}$. Its Fourier image is $\tau_{00}\left(p_{\alpha}\right)=2 \pi \mathcal{M} \delta\left(p_{0}\right)$, containing only tachyonic modes. Hence, we use the formulas of Sec. V.

We take into account (30) and (64) for the Fourier transform of the solution for the induced metric on the first brane with the source on the same brane. Collecting all expressions together, we obtain the Fourier image of the gravitational potential

$$
V\left(p_{\alpha}\right)=\frac{2 \pi \mathcal{M} \delta\left(p_{0}\right)}{3}\left[\frac{k}{A \vec{p}^{2}}-\frac{2}{M^{3}|\vec{p}|} \cdot f(|\vec{p}|)\right],
$$

where the function $f(p)$ denotes the second fraction in expression (64) taken at the position of the visible brane $\left(z=z_{0}\right)$ :

$$
f(p) \equiv \frac{C_{K}^{*} I_{2}\left(\left|z_{0}\right|\right)+C_{I}^{*} K_{2}\left(\left|z_{0}\right|\right)}{C_{I}^{*} C_{K}^{0}-C_{I}^{0} C_{K}^{*}} .
$$

The potential $V(r)$ is obtained by taking the inverse Fourier transform of (94):

$$
V(r)=-\frac{k \mathcal{M}}{3 \pi^{2} M^{3} r}\left[I(r)-\frac{\pi M^{3}}{4 A}\right],
$$

where

$$
I(r)=\int_{0}^{\infty} d s \sin (k r s) \Psi(s), \quad \Psi(s)=\frac{D(s)+\mu_{*} \alpha s D_{2}(s)}{E(s)}
$$

and $E(s)$ is given by Eq. (67).

The integral in (97) cannot be evaluated exactly, but it can be approximated in different regions of $r$, as it is done, e.g., in $[12,22]$.

1. On very large spatial scales $k r \gg \alpha \equiv e^{k \rho}$, we need the asymptotics of the function $\Psi(s)$ in the region $\alpha s \ll k r s \sim 1$. It is given by the expression

$$
\begin{aligned}
\Psi(s) & \sim \frac{2}{1+2 \mu+\alpha^{-2}\left(2 \mu_{*}-1\right)} \cdot \frac{1}{s} \\
& +\frac{\left(\alpha^{2}-1\right)\left(2 \mu_{*}-1\right)\left[3 \alpha^{2}-1+2 \mu_{*}\left(1-\alpha^{2}\right)\right]+4 \alpha^{2} \ln \alpha}{4\left[\alpha^{2}(1+2 \mu)+2 \mu_{*}-1\right]^{2}} \cdot s,
\end{aligned}
$$

and the integral in (97) is approximated by using the regularization $[12,22]$

$$
\int_{0}^{\infty} s \sin (k r s) d s \rightarrow \lim _{\epsilon \rightarrow 0} \int_{0}^{\infty} s \sin (k r s) e^{-\epsilon s} d s=0
$$

with the result 


$$
I(r) \approx \frac{\pi}{1+2 \mu+\alpha^{-2}\left(2 \mu_{*}-1\right)}=\text { const }
$$

The potential in this region has Newtonian form

$$
V(r)=-\frac{G \mathcal{M}}{r}, \quad G=G_{\mathrm{N}}\left(1+\frac{B}{3 A \alpha^{2}}\right),
$$

where $G_{\mathrm{N}}$ given by $(51)$, which is in complete agreement with the zero-mode approximation (49). The theory has continuous Newtonian limit as $\alpha \rightarrow \infty$.

2. On the scales $1 \ll k r \ll \alpha$, it is the region of integration $1 / \alpha \ll s \ll 1$ that substantially contributes to the integral in (97). In this region, we have

$$
\Psi(s) \approx \frac{1}{1+2 \mu} \cdot \frac{1}{s}-\frac{\log (s / 2)}{(1+2 \mu)^{2}} \cdot s,
$$

substituting which to (97), we obtain

$$
I(r)=\frac{\pi}{1+2 \mu}+\frac{\pi}{2(1+2 \mu)^{2}} \cdot \frac{1}{(k r)^{2}}
$$

and

$$
V(r)=-\frac{G \mathcal{M}}{r}\left(1+\frac{2 M^{3}}{3 A(k r)^{2}}\right), \quad G=G_{\mathrm{N}}\left(1-\frac{B}{A \alpha^{2}}\right)
$$

We observe corrections to the Newtonian potential similar to those of the RandallSundrum model [2] but with somewhat different relative constant.

3. In the case $k r \ll 1$, we can use the asymptotics for the function $\Psi(s)$ at infinity:

$$
\Psi(s) \sim \frac{1}{\mu s+1+15 \mu / 8}\left[1+\mathcal{O}\left(s^{-1}\right)\right], \quad \mu \neq 0 .
$$

This case is further partitioned into two asymptotic regions, depending on the magnitude of $\mu$.

3a. $k r \ll|\mu|$. Here, if $\mu$ is negative, then it cannot be small by absolute value since, in this latter case, the theory contains tachyons. We obtain

$$
I(r) \approx \frac{\pi}{2 \mu}+\frac{1}{\mu}\left(\frac{15}{8}+\frac{1}{\mu}\right) k r \log \left[\left(\frac{15}{8}+\frac{1}{\mu}\right) k r\right]
$$

and

$$
V(r)=-\frac{G \mathcal{M}}{r}-\frac{k \mathcal{M}}{3 \pi^{2} m^{2}}\left(\frac{15}{8}+\frac{1}{\mu}\right) \log \left[\left(\frac{15}{8}+\frac{1}{\mu}\right) k r\right], \quad G=\frac{1}{8 \pi m^{2}} \cdot \frac{\mu+2 / 3}{\mu+1 / 2} .
$$

The logarithmic corrections in these expressions assume that the expression $15 / 8+1 / \mu$ is not very small by absolute value.

3b. $\mu \ll k r \ll 1$. Here we must consider only positive $\mu$. In this case,

$$
I(r) \approx \frac{1}{k r}
$$


and the gravitational law is five-dimensional:

$$
V(r)=-\frac{\mathcal{M}}{3 \pi^{2} M^{3} r^{2}}\left(1-\frac{\pi}{4} k r\right) .
$$

The expressions for the gravitational potentials obtained in cases $3 \mathrm{a}$ and $3 \mathrm{~b}$ are analogous to those of [4]. The same results would be obtained in the linear approximation in the onebrane case considered in [13], as is clear from the remark made after Eq. (65), which identifies the corresponding propagators.

\section{B. Mater source on the hidden brane}

In a similar way one can consider the case where the stationary matter resides on the hidden brane with mass $\mathcal{M}_{*}$ defined as $\tau_{00}^{*}=\mathcal{M}_{*} \delta(\vec{r})$. In this case, we obtain the following expression for the gravitational potential on the visible brane:

$$
V(r)=-\frac{k \mathcal{M}_{*}}{3 \pi^{2} M^{3} \alpha r} I(r),
$$

where

$$
I(r)=\int_{0}^{\infty} d s \sin (k r s) \Psi(s), \quad \Psi(s)=\frac{I_{1}(s) K_{2}(s)+I_{2}(s) K_{1}(s)}{E(s)}
$$

and $E(s)$ is given by Eq. (67).

The asymptotic expressions for $\Psi(s)$ can be found in various regions:

$$
\begin{gathered}
\Psi(s) \approx \frac{2 \alpha}{2 \mu_{*}-1+\alpha^{2}(2 \mu+1)} \cdot \frac{1}{s}+\mathcal{O}(s), \quad s \ll \alpha^{-1}, \\
\Psi(s) \approx \frac{\sqrt{2 \pi} e^{-\alpha s}}{(1+2 \mu) \mu_{*} \sqrt{\alpha s}}\left[1+\left(\frac{15}{8}-\frac{1}{\mu_{*}}\right) \frac{1}{\alpha s}+\mathcal{O}\left(s^{2}\right)\right], \quad \alpha^{-1} \ll s \ll 1, \quad \mu_{*} \neq 0, \\
\Psi(s) \approx \frac{\sqrt{2 \pi \alpha s} e^{-\alpha s}}{(1+2 \mu)}\left[1+\frac{3}{8 \alpha s}+\mathcal{O}\left(s^{2}\right)\right], \quad \alpha^{-1} \ll s \ll 1, \quad \mu_{*}=0, \\
\Psi(s) \approx \frac{2 \sqrt{\alpha} e^{-\alpha s}}{\mu \mu_{*} \alpha s^{2}+\left(\mu+\mu_{*} \alpha+15 \mu \mu_{*} \alpha / 8\right) s}, \quad s \gg 1, \quad \mu^{2}+\mu_{*}^{2} \neq 0, \\
\Psi(s) \approx 2 \sqrt{\alpha} e^{-\alpha s}, \quad s \gg 1, \quad \mu=\mu_{*}=0 .
\end{gathered}
$$

Using these expression, it is not difficult to obtain the estimates for the gravitational potential $V(r)$ caused by the presence of the static source on the hidden brane in various regions. We have 


$$
V(r) \approx-\frac{4 G_{\mathrm{N}} \mathcal{M}_{*}}{3 \alpha^{2} r}, \quad k r \gg \alpha
$$

where $G_{\mathrm{N}}$ is given by (51). Again, in this distance range, the result can be obtained by using the zero-mode approximation (52).

In the case $k r \ll \alpha$, the result crucially depends on whether $\mu_{*}$ is zero or not:

$$
\begin{gathered}
V(r) \approx-\frac{c_{1} k^{2} \mathcal{M}_{*}}{\pi^{2} A \alpha^{3}}\left[1-c_{2}\left(\frac{k r}{\alpha}\right)^{2}\right], \quad k r \ll \alpha, \quad \mu_{*}=0, \\
V(r) \approx-\frac{2 k^{2} \mathcal{M}_{*}}{3 \pi^{2} A \alpha^{3}}\left[\left(1+\frac{c_{3}}{\mu_{*}}-\frac{c_{4}}{\mu_{*}^{2}}\right)-\left(c_{5}+\frac{c_{6}}{\mu_{*}}-\frac{c_{7}}{\mu_{*}^{2}}\right) \cdot\left(\frac{k r}{\alpha}\right)^{2}\right], \quad k r \ll \alpha, \quad \mu_{*} \neq 0,
\end{gathered}
$$

Here the constants $c_{n}$ take the following approximate values:

$$
c_{1} \approx 1.77, \quad c_{2} \approx 1.02, \quad c_{3} \approx 1.3, \quad c_{4} \approx 0.35, \quad c_{5} \approx 0.06, \quad c_{6} \approx 1.12, \quad c_{7} \approx 0.24 .
$$

Expression (119) is not valid for sufficiently small $\mu_{*}$ since we know that, in the limit of $\mu_{*} \rightarrow 0$, the asymptotics changes to (118). In fact, comparison with the exact numerical integration of (111) shows that our approximate result (119) is only good for $\left|\mu_{*}\right| \gtrsim 0.5$.

\section{DISCUSSION}

It is known that the braneworld model becomes rather rich in its cosmological manifestations if curvature term is present in the action for the brane (see $[4-6,12,14-16]$ ). In this paper, we studied the model with scalar-curvature terms for the branes on the original Randall-Sundrum two-brane background. The linearized gravitational equations (49) and (52) in this case have the same structure as in the original Randall-Sundrum model but with different physical constants. In the limit of vanishing brane Planck masses $m$ and $m_{*}$, they tend to the known results $[12,20,22]$, which are physically reasonable only for the bulk Planck mass $M>0$. In the opposite limit of $M \rightarrow 0$ while $k=\sigma / M^{3}$ is fixed, they produce reasonable results (55) and (56) independently of the sign of $M$.

In this paper, we developed a general method for detecting tachyonic modes in the braneworld theory, which can be generalized to theories giving rise to equations of the similar kind, e.g., the theory with Gauss-Bonnet term in the bulk action [8]. In our case, for negative values of $M$, the linearized theory typically contains tachyonic modes in the gravitational sector. If both brane Planck masses are nonzero, then we have one or two tachyonic mass eigenvalues depending on the constants of the theory, the conditions of which were determined in Sec. V. However, in the case where one of the brane Planck masses is zero, tachyonic modes are absent if the other brane has sufficiently high Planck mass [given by Eq. (79) for the visible brane, and by Eq. (83) for the hidden brane]. In the case of negative bulk Planck mass $M$, the zero-mode graviton is 'localized' around the 
brane with negative tension and is not a ghost in all cases where tachyonic modes are absent in the theory. However, in all cases with negative $M$, the massive gravitational modes of the theory under consideration have ghost-like character. The conditions of absence of both radion ghosts and gravitational tachyons are expressed by (85).

Exploring both signs of the bulk gravitational constant may be interesting in connection with some braneworld cosmological models requiring negative brane tension, such as the model of disappearing dark energy (DDE) recently discussed in [14,15] or the braneworld models with 'quiescent' cosmological singularities during expansion [16]. The DDE model $[14,15]$ represents a cosmological braneworld with the Randall-Sundrum constraint (2), negative brane tension, and the condition $|\mu| \geq 1 / 2$, which is required for physical consistency and which implies inequality (79). The 'quiescent' singularities in the AdS-embedded braneworld models occur during the universe expansion and are characterized by finiteness of the scale factor, Hubble parameter, and matter density. The braneworld cosmological equations involve the bulk gravitational constant only in even power; therefore, their behaviour is independent of its sign on the homogeneous and isotropic level [14]. However, as noted in the introduction, the sign of the bulk gravitational constant is of importance for the small-scale gravitational physics in a braneworld universe, in particular, for the behaviour of cosmological perturbations. The results of the present paper indicate that models with negative bulk gravitational constant can be free from tachyons, although they are plagued with massive ghosts in the gravitational sector. Perhaps, the unwanted situation with ghosts can be remedied by modifications of the bulk action. It should be emphasized that these results do not relate to the braneworld cosmological models with negative brane tension but positive bulk and brane gravitational constants, which require future investigation.

\section{ACKNOWLEDGMENTS}

The authors are grateful to Varun Sahni for valuable comments and suggestions. Yu. S. acknowledges warm hospitality of the Inter-University Centre for Astronomy and Astrophysics (IUCAA) in Pune, India.

[1] Randall L and Sundrum R 1999 Phys. Rev. Lett. 833370 (Preprint hep-ph/9905221)

[2] Randall L and Sundrum R 1999 Phys. Rev. Lett. 834690 (Preprint hep-th/9906064)

[3] Deruelle N 2003 Astrophys. Space Sci. 283619 (Preprint gr-qc/0301035) Maartens R 2003 Prog. Theor. Phys. Suppl. 148213 (Preprint gr-qc/0304089)

[4] Dvali G, Gabadadze G and Porrati M 2000 Phys. Lett. B 485208 (Preprint hep-th/0005016) Dvali G and Gabadadze G 2001 Phys. Rev. D 63065007 (Preprint hep-th/0008054)

[5] Collins H and Holdom B 2000 Phys. Rev. D 62105009 (Preprint hep-ph/0003173) Collins H and Holdom B 2000 Phys. Rev. D 62124008 (Preprint hep-th/0006158) Shtanov Yu V 2000 On brane-world cosmology, (Preprint hep-th/0005193)

[6] Deffayet C 2001 Phys. Lett. B 502199 (Preprint hep-th/0010186) 
Deffayet C, Dvali G and Gabadadze G 2002 Phys. Rev. D 65044023 (Preprint astroph/0105068)

[7] Davoudiasl H, Hewett J L and Rizzo T G 2003 JHEP 0308034 (Preprint hep-ph/0305086)

Dubovsky S L and Libanov M V 2003 JHEP 0311038 (Preprint hep-th/0309131)

[8] Charmousis C and Dufaux J-F 2004 Phys. Rev. D 70106002 (Preprint hep-th/0311267)

[9] Padilla A 2004 Class. Quantum Grav. 212899 (Preprint hep-th/0402079)

[10] Smolyakov M N 2004 Nucl. Phys. B 695301 (Preprint hep-th/0403034)

[11] Shtanov Yu and Viznyuk A 2004 Mirror branes, (Preprint hep-th/0404077)

[12] Kiritsis E, Tetradis N and Tomaras T N 2002 JHEP 0203019 (Preprint hep-th/0202037)

[13] Tanaka T 2004 Phys. Rev. D 69024001 (Preprint gr-qc/0305031)

[14] Sahni V and Shtanov Yu 2003 JCAP 0311014 (Preprint astro-ph/0202346)

Sahni V and Shtanov Yu 2002 Int. J. Mod. Phys. D 111515 (Preprint gr-qc/0205111)

[15] Alam U and Sahni V 2002 Supernova constraints on braneworld dark energy, (Preprint astro$\mathrm{ph} / 0209443)$

[16] Shtanov Yu and Sahni V 2002 Class. Quantum Grav. 19 L101 (Preprint gr-qc/0204040)

[17] Birrell N D and Davies P C W 1982 Quantum Fields in Curved Space (Cambridge: Cambridge University Press)

[18] Shtanov Yu V 2002 Phys. Lett. B 543121 (Preprint hep-th/0108211)

[19] Garriga J and Tanaka T 2000 Phys. Rev. Lett. 842778 (Preprint hep-th/9911055)

Charmousis C, Gregory R and Rubakov V A 2000 Phys. Rev. D 62067505 (Preprint hepth/9912160)

Barvinsky A O, Kamenshchik A Yu, Rathke A and Kiefer C 2003 Phys. Rev. D 67023513 (Preprint hep-th/0206188)

[20] Smolyakov M N and Volobuev I P 2002 Linearized gravity, Newtonian limit and light deflection in RS1 model, (Preprint NPI MSU 2002-18/702, hep-th/0208025)

[21] Shiromizu T, Maeda K and Sasaki M 2001 Phys. Rev. D 62024012 (Preprint hep-th/9910076)

[22] Jung E, Kim S H and Park D K 2003 Nucl. Phys. B 669306 (Preprint hep-th/0305156) 